\title{
Comparative significance of pretectal and ventral lateral geniculate systems in functional recoveries after injuries to the posterior cortex
}

\author{
THACKERY S. GRAY, DAVID G. LAVOND, PATRICIA M. MEYER \\ and DONALD R. MEYER \\ Laboratory of Comparative and Physiological Psychology, Ohio State University \\ Columbus, Ohio 43212
}

\begin{abstract}
Rats with ablations of the pretectal region or ventral lateral geniculate bodies rapidly relearn a black-white discrimination. However, if combined with visual cortical ablations, pretectal ablations, but not LGNv ablations, markedly potentiate impairments of retention of the task. The results are discussed in relation to the putative role of sprouting in recoveries of functions.
\end{abstract}

Lashley (1935) observed that rats which were trained on a black-white discrimination problem and were subsequently subjected to lesions of the posterior cortex failed to exhibit postoperative retention although they could relearn the problem. Later, Horel, Bettinger, Royce, and Meyer (1966) established that subjects which were trained with first-stage anterior neocortical ablations and were then retrained after second-stage posterior ablations relearned the blackwhite problem just as rapidly as rats with only posterior neocortical lesions. Also, Meyer, Yutzey, Dalby, and Meyer (1968) demonstrated that one-stage removals of the entire neocortex did not prevent relearning of the discrimination. Hence, relearning of the black-white problem is independent of the neocortex and consequently must be mediated by mechanisms located at a subcortical level.

The failure of the posteriorly decorticated rat to exhibit retention of the black-white problem cannot be explained as a loss of the associative substrates or engrams which are formed preoperatively, but rather it appears to be due to an impairment of retrieval (Meyer, 1972). Thus, Braun, Meyer, and Meyer (1966) found that injections of amphetamine facilitate recovery of a preoperatively learned blackwhite habit in rats with lesions of the visual cortex. However, naive bilateral posterior preparations treated with amphetamine learned the habit at about the same rate as normal control animals. Since amphetamine treatments facilitated relearning, but not original learning, the finding indicated that the

This research was supported, in part, by Grant MH-06211 from the National Institute of Mental Health to Donald R. Meyer and Patricia M. Meyer. The authors wish to thank Mary G. Hata for her assistance in this investigation. engrams established in rats prior to surgery were not completely destroyed. This result was later replicated by Jonason, Lauber, Robbins, Meyer, and Meyer (1970).

Additional support for the concept of impaired retrieval was provided by a study of LeVere and Morlock (1973). Those investigators trained rats on the black-white problem, removed the posterior neocortex, and then retrained the subjects on the same problem or on a reversal of the problem. Their significant finding was that rats that were retrained on the reversals relearned the problem much more slowly than rats that were retrained on the identical preoperatively learned discriminations. Accordingly, the outcome indicated that the preoperative engram was intact, and that its presence could only be detected through the use of an interference paradigm.

The foregoing facts are all consistent with the notion that training on black-white problems leads to the formation of subcortical as well as cortical retrieval mechanisms. What is not clear is why the subcortical systems will not ordinarily support postoperative performance of the problem following destruction of posterior neocortex. Nor has it been established for certain which neural systems are involved in the mediations of the postoperative recovery or whether these systems contribute differentially to the process of the recovery.

As part of the program that is aimed at the solution of these problems, we studied the effects of lesions to the pretectal area (PT) and the ventral lateral geniculate nuclei (LGNv) in animals with and without the posterior neocortex. These areas are of interest because they receive collateral sprouts from the optic tract (OT) in response to posterior cortical ablations (Cunningham, 1972; Goodman \& Horel, 
1966), but as yet there is no proof that the sprouted pathways contribute to the process of recovery of performance of the black-white discrimination problem. Our first aim was to determine the extent to which the LGNv and PT contributed to the recovery of the problem. Then we attempted to ascertain the pattern of recovery in preparations which eliminated the possibility of OT sprouting to these subcortical sites, that is, in animals with posterior neocortical ablations combined with LGNv and or PT lesions.

\section{METHODS}

\section{Subjects}

The study was conducted with 211 Long-Evans hooded rats which were 90-120 days of age at the beginning of training. They were housed in separate cages and were maintained on an ad-lib food and water schedule.

\section{Behavioral Apparatus and Procedures}

The animals were trained on a black-white discrimination problem in the Thompson-Bryant (1955) apparatus. The apparatus consisted of a startbox, a choice compartment, and a goal compartment that was separated from the choice compartment by a vertical partition. Two square openings within the partition were fitted with black and white stimulus doors whose luminances were approximately 0.1 and $3.0 \mathrm{~mL}$. The walls of the startbox, choice compartment, and goalbox were constructed of the same black plastic material as the wall that held the stimulus panels. The apparatus was illuminated by small fluorescent lamps which were mounted on the transparent lids of the start and choice compartments.

The training procedures were the same as those employed by Glendenning (1972). Prior to pretraining, each of the rats was handled for at least $5 \mathrm{~min}$ per day for a total of 5 days. Then, on Day 6, each rat was permitted to explore the entire apparatus for $15 \mathrm{~min}$. Then, on Day 7, a guillotine door was inserted between the startbox and the choice compartment, and the animal was trained to escape shock which was delivered through a floor grid by running into the choice compartment and into the goalbox when the guillotine door was raised. Pretraining doors were made of black and white stripes oriented obliquely and then placed into the doorways between the choice compartment and the goalbox; the rat was trained to enter the goalbox by knocking down a pretraining door. On Day 8 , the black and white training doors were put into the doorways, and the rat was trained to enter the goalbox by knocking down the unlocked white door. The left-right positions of the two stimuli were governed by a Gellerman (1933) series, and the animals were trained for 25 trials per day, with an intertrial interval of approximately $90 \mathrm{sec}$, until they had reached a criterion of 9 correct responses in 10 trials.

\section{Experimental Design}

When preoperative training on the black-white discrimination problem was complete, the animals were individually assigned to one of seven groups. The groups were matched in terms of the initial learning scores of the members. All surgeries were performed on the day following initial acquisition of the habit. One group of rats was subjected to bilateral ablations of the LGNv, another to bilateral ablations of PT, and a third group to bilateral removals of both PT and LGNv (PT-LGNv). The fourth group received simultaneous bilateral ablations of $\mathrm{PT}$ and the posterior neocortex (PT-VC), the fifth underwent removals of LGNv and VC (LGNv-VC), and the sixth received lesions of PT, LGNv, and VC (PT-LGNv-VC). The final group was subjected to bilateral removal of posterior neocortex (VC). After a 12-day recovery interval, the animals were retrained on the same black-white task to the same preoperative criterion.

\section{Surgical Procedures}

The surgery was performed with clean technique while the rats were anesthetized with sodium pentobarbital. The VC ablations were accomplished by the pia-stripping method (cf. Meyer \& Meyer, 1971). The lesions of intent extended from bregma to the posteror margins of the hemispheres and from the longitudinal fissure to the rhinal sulci. The ablations were characterized as radical because they were not restricted to the striate and the extra-striate cortex as defined by Krieg (1946). Thus they included all the zones of termination of projections from the dorsal lateral geniculate bodies and the nucleus lateralis posterior, namely, Krieg's areas 17, 18, 18A, 7, and 20 (cf. Hughes, 1977). The relationships between the foregoing zones and the extent of the radical lesion of intent have been described by Meyer and Meyer (1977, Figure 2).

The subcortical lesions were made via stereotaxic methods. The parameters were chosen on the basis of results obtained from 27 pilot subjects. Three landmarks, stereotaxic zero, the locus of the sagittal suture, and the midpoint between the dorsolateral skull ridges, were used in calculating the mediolateral (ML) zero. If any two of these three measurements agreed, that value was used as midline zero; otherwise ML zero was determined by using the average of the dorsolateral skull ridges. The de Groot (1959) coordinates for the LGNv lesions were $+3.0 \mathrm{~mm} \mathrm{AP}, \pm 4.2 \mathrm{~mm}$ $\mathrm{ML}$, and $+1.2 \mathrm{~mm} \mathrm{DV}$. The coordinates for the PT ablations were $+2.3 \mathrm{~mm} \mathrm{AP}, \pm 1.3 \mathrm{~mm} \mathrm{ML}$, and $+.5 \mathrm{~mm} \mathrm{DV}$. The lesions were produced by the passage of a $0.6-\mathrm{mA}$ anodal current through a No. 1 insect pin for $3 \mathrm{sec}$. The pin was insulated with a plastic coating except at the head and .2 to $.5 \mathrm{~mm}$ at the tip.

\section{Histological Procedures}

When postoperative testing was complete, each rat was administered a lethal dose of sodium pentobarbital and then perfused with normal saline followed by $10 \%$ Formalin. The brain was immediately removed and placed in $10 \%$ Formalin. After the extent of cortical damage was plotted on standard Lashley brain diagrams, the tissue was frozen and sectioned at $30 \mu$. Every fifth section through the dorsal thalamus of subjects with VC ablations and through the extent of the lesion in animals with subcortical injuries were saved, mounted on slides, and then stained with cresyl violet. Subsequently, the amount of retrograde degeneration in the LGNd was assessed. The extents of the subcortical surgeries were estimated by two independent observers, and drawings were made of the sections which contained the most extensive damage.

\section{Anatomical Results}

\section{RESULTS}

Figures 1 through 4 present the surface reconstructions of the brains for the 99 subjects which sustained VC ablations (29 in Group VC, 27 in LGNv-VC, and 24 in PT-LGNv-VC). Also included in the figures above the surface diagrams are estimates of the amount of retrograde degeneration in the respective LGNd. Principal cells were absent in 88 cases. There were 7 animals with localized sparing in Group VC; of these, 5 were unilateral and 2 were bilateral. There were 2 instances of unilateral sparing in Group LGNv-VC. The other two rats with localized sparing were in the PT-LGNv-VC group; one was unilateral and the other was bilateral. 


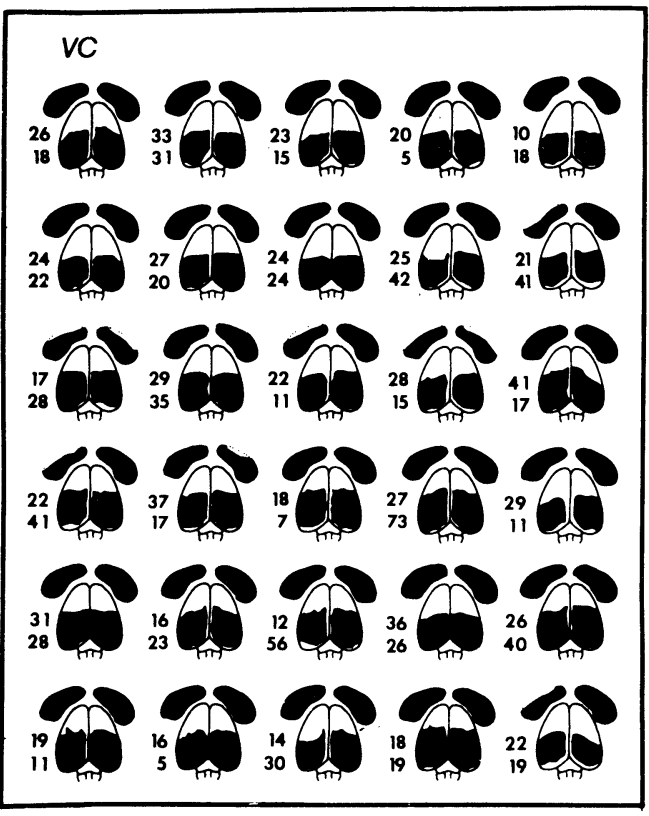

Figure 1. Surface diagrams of posterior neocortical ablations and representations of degeneration in the respective LGNds of Group VC.

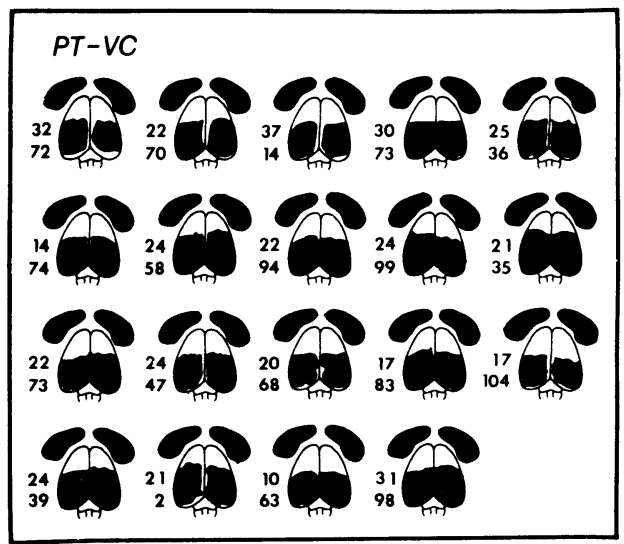

Figure 2. Surface diagrams of posterior neocortical ablations and representations of degeneration in the respective LGNds of Group PT-VC.

The reconstructions of the subcortical lesions are shown in Figures 5 through 11. The LGNv ablations were judged to be acceptable (hits) provided that the lesions were complete and bilaterally symmetrical. The criteria for accepting the PT ablations as hits were bilateral destructions of the lateral nuclei of the OT and the nuclei pretectalis, the regions in Goodman and Horel (1966) reported collateral optic tract sprouting. Also, there could be no involvement of the superficial layers of superior colliculi or the lateral

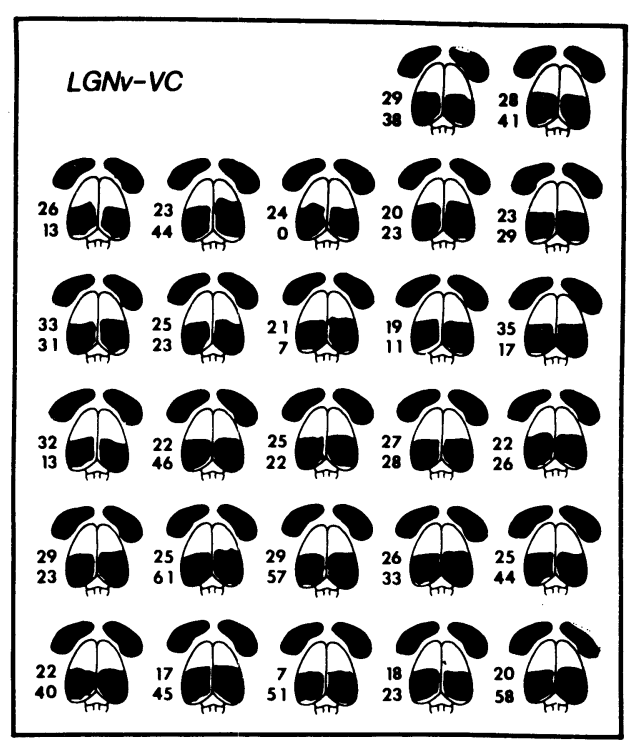

Figure 3. Surface diagrams of posterior neocortical ablations and representations of degeneration in the respective LGNds of Group LGNv-VC.

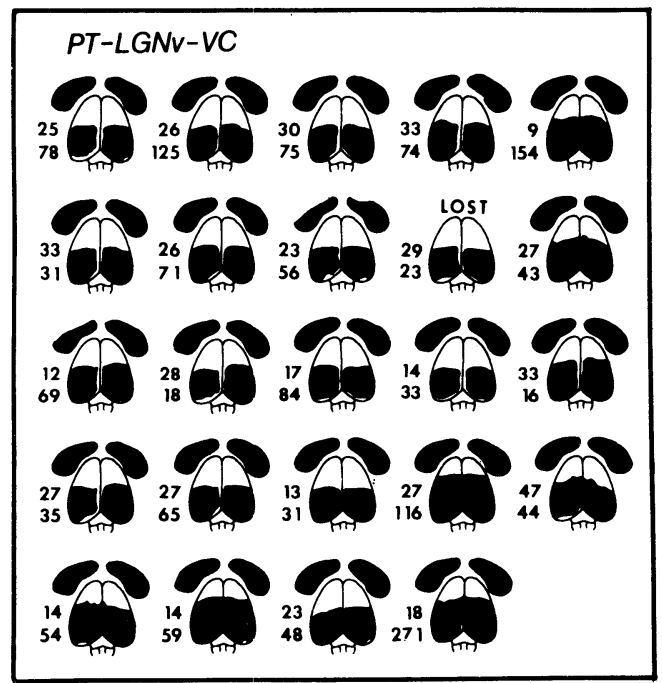

Figure 4. Surface diagrams of posterior neocortical ablations and representations of degeneration in the respective LGNds of Group PT-LGNv-VC.

posterior nuclei (NLP) except that encroachments upon NLP were accepted when a PT ablation was combined with a VC ablation. Of the 154 attempted ablations of the LGNv or PT, 90 were classified as hits and 64 as misses.

\section{Behavioral Results}

The trials required by the individual subjects to learn and to relearn the black-white problem are given with the brain diagrams in Figures 1 through 


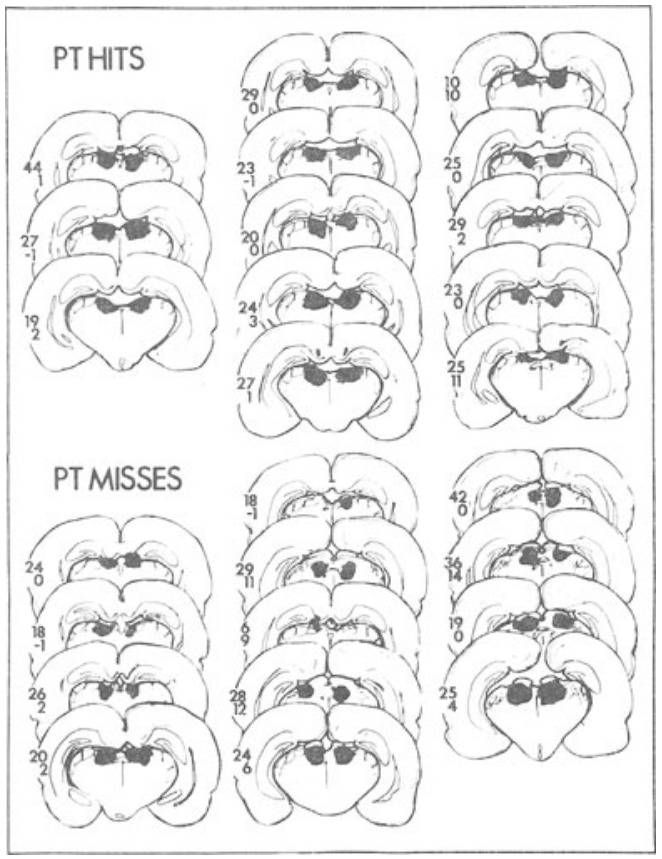

Figure 5. Representative subcortical PT lesions for Group PT hits and misses.

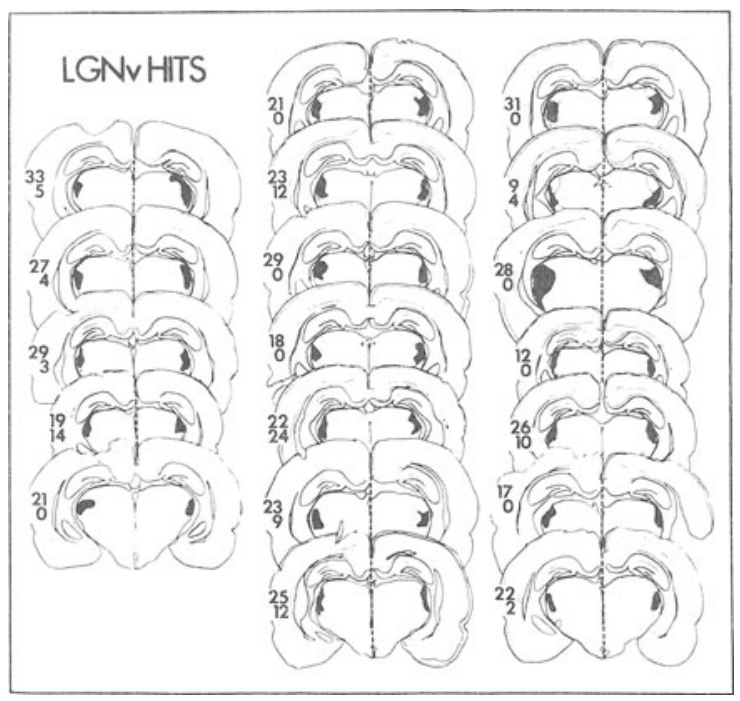

Figure 6. Representative subcortical LGNv lesions for Group LGNv hits.

11. The learning scores are given at the top, and the relearning scores at the bottom. Table 1 presents the group means, the standard errors of the means, and the medians for trials which were required for postoperative relearning of the problem by the various groups and subgroups.

It is apparent that the rats that were prepared with LGNv or PT ablations retained the habit very well. Indeed, half the subjects of the PT group relearned the problem in one trial or less, and the same thing was true of 13 of the 34 subjects in the LGNv group. The results for the subgroups of both groups were also essentially the same, and the intergroup differences of three trials or less were obviously not significant.

The subjects with PT-LGNv ablations which were classified as hits required about $13 / 10$ mean/median trials to relearn the problem. The subjects with PTLGNv ablations which were classified as misses also required 10 median trials to relearn the problem, but their behaviors were inconsistent, as is clear from

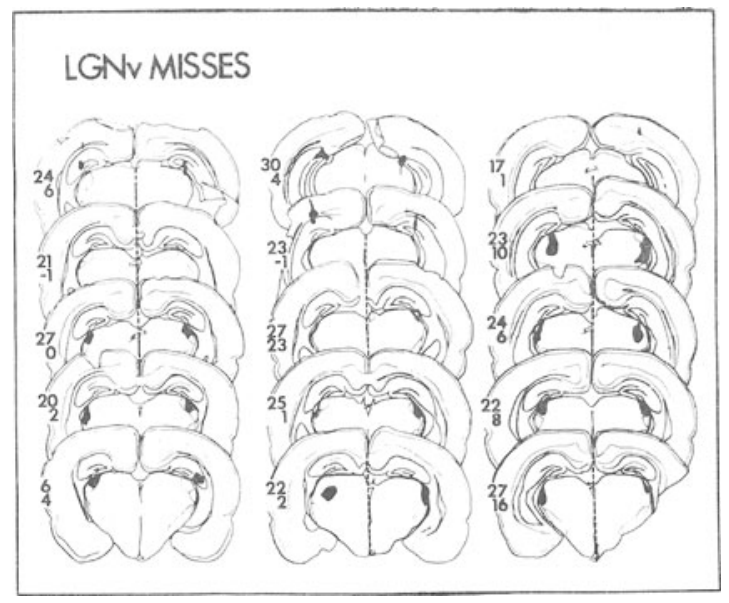

Figure 7. Representative subcortical LGNv lesions for Group LGNv misses.

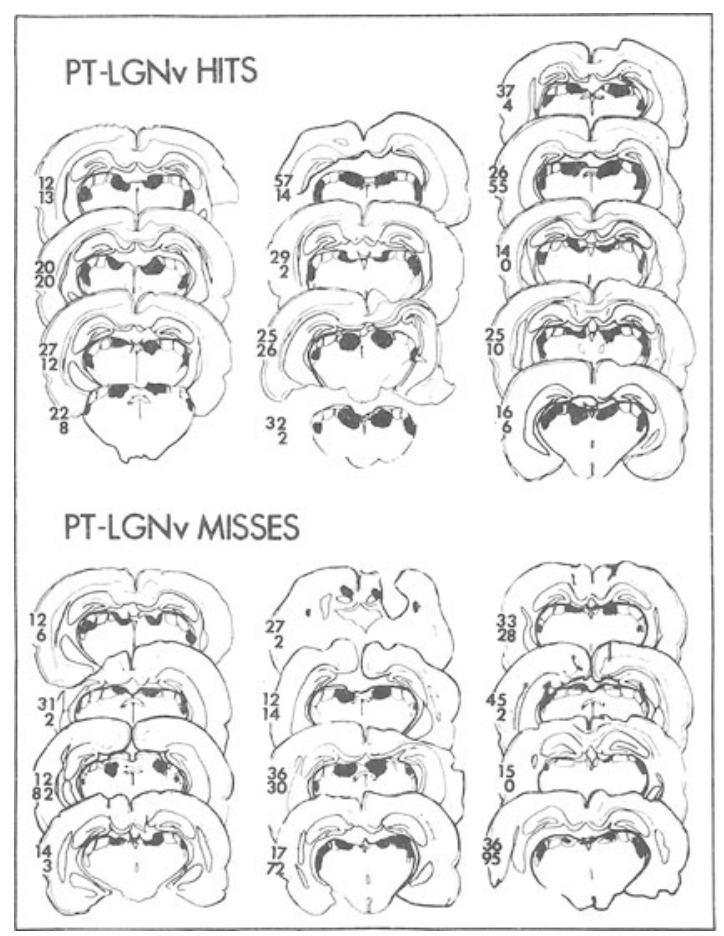

Figure 8. Representative subcortical PT and LGNv lesions for Group PT-LGNv hits and misses. 


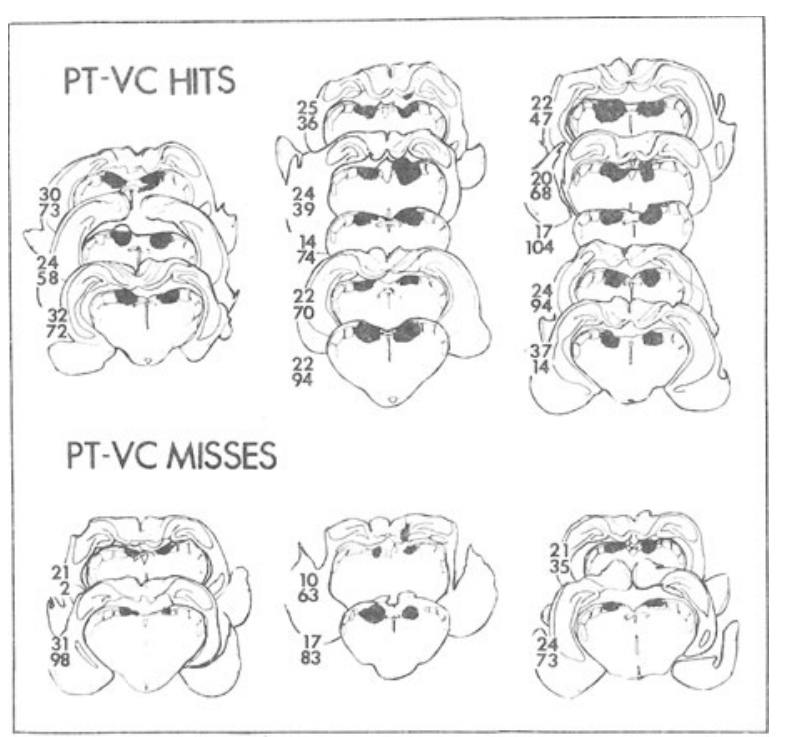

Figure 9. Representative subcortical PT and VC lesions for Group PT-VC hits and misses.

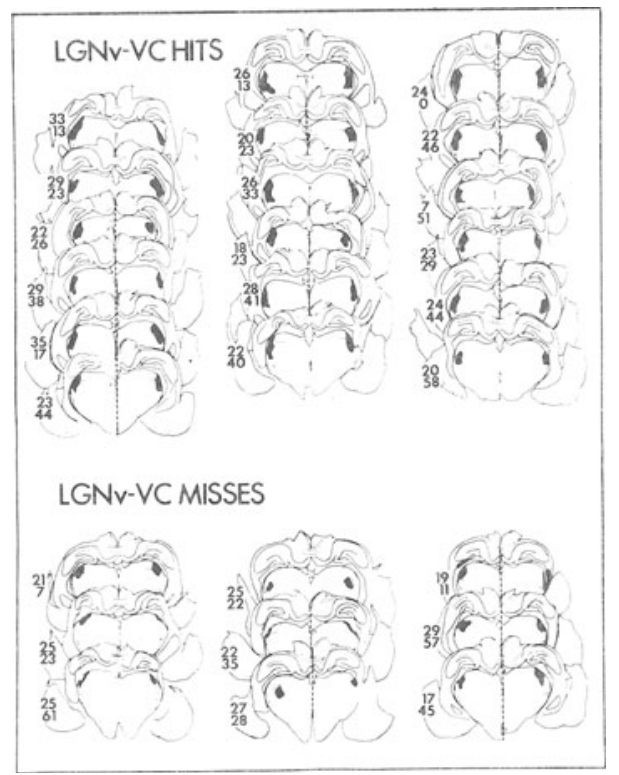

Figure 10. Representative subcortical LGNv and VC lesions for Group LGNv-VC hits and misses. the fact that their mean score was high and the standard error was relatively large.

The 30 animals with VC ablations relearned the problem in approximately 25 mean trials. The result was as expected from the findings of Glendenning (1972), whose 15 subjects with VC ablations relearned the problem in approximately 23 mean trials (cf. Figure 14, Meyer \& Meyer, 1977). The performances of the hit and miss VC subgroups, which were formed on the basis of whether the ablations resulted in complete or incomplete degeneration of the LGNds, differed by less than one mean trial and by only 3 median trials. The outcome confirmed a previous observation by Horel et al. (1966) that posterior ablations will suppress postoperative performance of the problem regardless of whether the LGNds still contain principal cells in the dorsolateral crescents.

The subjects of the LGNv-VC subgroup whose lesions were classified as hits relearned the problem in approximately $31 / 31$ mean/median trials. Both of

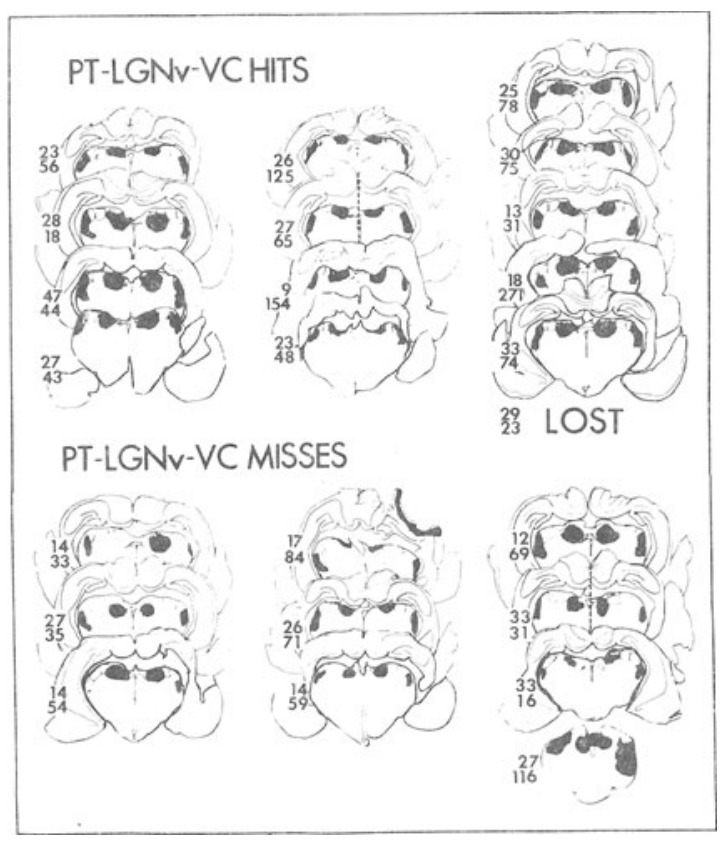

Figure 11. Representative subcortical PT, LGNv, and VC lesions for Group PT-LGNv-VC hits and misses.

Table 1

Mean/Median Relearning Scores for the Hits, Misses, and Combined Hits and Misses for All Seven Groups

\begin{tabular}{|c|c|c|c|c|c|c|c|c|c|c|}
\hline \multirow[b]{2}{*}{ Group } & \multirow[b]{2}{*}{$\mathrm{n}$} & \multicolumn{3}{|c|}{ Hits } & \multicolumn{3}{|c|}{ Misses } & \multicolumn{3}{|c|}{ Combined } \\
\hline & & Mean & Error & Median & Mean & Error & Median & Mean & Error & Median \\
\hline PT & 26 & 2.2 & 1.1 & 1.0 & 4.5 & 1.5 & 2.0 & 3.3 & .9 & 1.6 \\
\hline LGNv & 34 & 5.2 & 1.5 & 3.0 & 5.4 & 1.7 & 4.0 & 5.3 & 1.1 & 3.8 \\
\hline PT-LGNv & 25 & 13.2 & 4.0 & 10.0 & 28.1 & 10.1 & 10.0 & 20.3 & 5.4 & 10.0 \\
\hline $\mathrm{VC}$ & 30 & 25.0 & 3.4 & 23.0 & 24.6 & 4.7 & 19.0 & 24.9 & 2.8 & 21.0 \\
\hline LGNv-VC & 27 & 31.2 & 3.6 & 31.0 & 32.1 & 6.4 & 28.0 & 31.5 & 3.1 & 29.0 \\
\hline PT-VC & 19 & 64.9 & 7.1 & 70.0 & 59.0 & 14.3 & 68.0 & 63.0 & 6.4 & 70.0 \\
\hline PT-LGNv-VC & 24 & 78.9 & 17.9 & 60.5 & 56.8 & 9.4 & 55.5 & 69.7 & 11.2 & 57.5 \\
\hline
\end{tabular}


those scores were within three trials of the scores of the LGNv-VC subjects whose lesions were classified as misses, and hence the subgroups were combined and compared with the combined group of VC subjects. The scores for the LGNv-VC group were higher by approximately 7 mean and 8 median trials, but the intergroup difference was not significant as assessed by a Mann-Whitney $U$ test.

In contrast, the subjects with combined ablations of the PT and VC were very slow to relearn the problem. Thus, the mean and median relearning scores for the hit subgroup of the PT-VC group were more than twice and almost three times higher than the scores for the hit subgroup of the VC group. The subjects whose lesions were classified as misses required about twice as many mean/median trials to relearn the problem as the subjects of the VC miss group, and hence variations in the placements of the lesions appeared to be of little consequence. A Mann-Whitney $U$ test was used to compare the postoperative performance of the VC and PT-VC subjects, and the difference was significant beyond the .01 level.

As had been the circumstances for subjects with combined ablations of PT and LGNv, the results for the PT-LGNv-VC subjects were erratic. The hit and miss subgroups could not be distinguished from each other with a Mann-Whitney $U$ test and hence the subgroups were combined and compared with the combined PT-VC group. The mean performance of the latter group was lower than the mean performance of the PT-LGNv-VC subjects, but the opposite was true for the two groups' median scores. Hence, it could not be established that ablations of the LGNv potentiate the deficits of either VC or PT-VC preparations, which left the PT-VC result as the principal outcome of the study.

Figure 12 presents the behavioral outcomes for the seven groups of subjects in terms of the mean trials required by the animals to relearn the problem to successively more stringent criteria of postoperative performance. The functions were constructed by the method of Melton (1936) and as described by Meyer and Meyer (1977). The functions are based upon the data for all of the subjects within the various groups because, as we have noted, the subgroups' performances could not be distinguished from each other.

Finally, we asked if there were any relationships between the learning and relearning scores for the subjects within the various groups. Pearson coefficients for LGNv, PT, VC, PT-LGNv, LGNv-VC, PT-VC, and PT-LGNv-VC subjects were $.02, .00$, $.00, .00, .05, .04$, and .08 , respectively. The result confirms the outcomes of previous studies of the question; these have been reviewed by Jonason et al. (1970). As a group, the outcomes suggest once again that there is simply no basis for the use of savings scores as measures of postoperative recoveries of performance of the black-white discrimination problem.

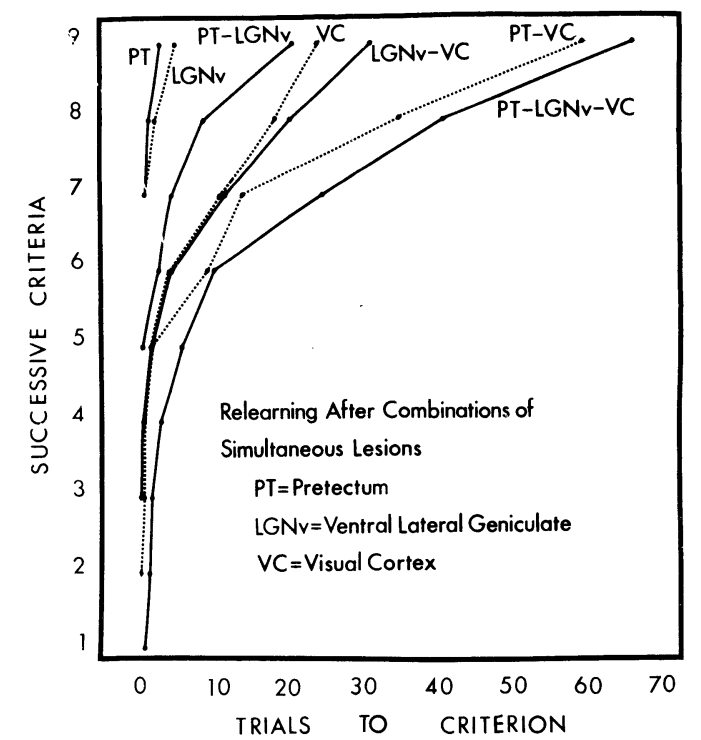

Figure 12. Mean trials to relearn the problem to successively more stringent criteria.

\section{DISCUSSION}

If rats are prepared with PT ablations or ablations of the LGNv, they exhibit no postoperative impairment of performance of the black-white problem. Our findings with respect to PT ablations are consistent with the observations of Blochert, Ferrier, and Cooper (1976) for the rat and of Hodos and Bonbright (1975) for the pigeon. They differ from the findings of Thompson and Rich (1963), but the difference is interpretable in terms of the fact that the lesions of the subjects of their investigation were much more extensive and included the nucleus posterior thalami (NPT) as well as the pretectal region. Thompson and Rich believed that the impairments of retention that they noted were primarily produced by the lesion of the NPT, and the present observations suggest that their conclusion was correct.

Our failures to observe significant impairments as a consequence of lesions of the LGNv are also in keeping with a finding of Hodos and Bonbright (1975). However, they were not as expected from the study of Horel (1968), who found that ablations of the LGNv bring about substantial retentive deficits as measured by the savings method. The difference was possibly a function of the fact that Horel's ablations were significantly larger than ours, and also included portions of the superior thalamic radiations and the thalamic reticular nucleus. But Horel's rats were also behaviorally peculiar in that the animals' preoperative scores were very much lower than the scores for his other groups of subjects. Hence, it is possible to think that the subjects had been undertrained before surgery, and if so that their seeming deficits were due, in large part at least, to forgetting. 
Our findings with respect to the effects of ablations of the LGNv are also at variance with the outcomes of a recent experiment of Legg and Cowey (1977). As to the reasons for the difference, perhaps it might have been a function of their having employed an unusually strict criterion of 36 out of 40 correct responses. Furthermore, as their data is only presented in savings scores and errors to criteria, we converted several of our animals' postoperative scores in trials to criteria to savings scores. In our sample of 19 subjects with lesions classified as hits, four of the animals had savings scores of $-.09, .26, .48$, and .52 . These values overlap with the savings scores of $-.28,-.14, .22$, and .33 of the four subjects which were studied by Legg and Cowey, and hence it is possible to think that their results could have well have been different had they studied a somewhat larger group.

Our most important finding is that PT ablations, if simultaneously combined with ablations of the VC, produce a much greater retardation of relearning than simultaneously combined ablations of the VC and LGNv. We know of no other comparable results. However, Horel (1968) studied preparations which were first subjected to VC ablations, were trained on the problem, and were subsequently tested for performance following a second-stage LGNv or PT ablation. Horel observed that the animals with second-stage LGNv lesions relearned the problem in about two-thirds as many trials as the subjects with the second-stage PT ablations. Hence, although the difference he observed was much smaller than the difference found in this study, both sets of data are supportive of the concept that recoveries of performance of the black-white problem following a VC ablation depend upon PT-related mechanisms to a greater extent than systems related to the LGNv.

It is clear from our findings with respect to the effects of PT-LGNv-VC ablations that systems apart from those considered in this study can mediate recoveries of performance of the problem by posteriorly decorticated subjects. However, it is equally clear that the cost to a VC subject of a PT ablation is substantially greater than the cost to such a subject of an injury to the LGNv. Accordingly, we think that it will next be of interest to ask if there are comparable differences between the patterns of sprouting which are found within these subjects after cortex is destroyed (Goodman \& Horel, 1966). As yet, we have no answer to the question, but we do have experiments in progress.

\section{REFERENCES}

Blochert, P. K., Ferrier, R. J., \& Cooper, R. M. Effects of pretectal lesions on rats wearing light-diffusing occluders. Brain Research, 1976, 104, 121-128.

Braun, J. J., Meyer, P. M., \& Meyer, D. R. Sparing of a bright- ness habit in rats following visual decortication. Journal of Comparative and Physiological Psychology, 1966, 61, 79-82.

Cunningham, T. J. Sprouting optic projection after cortical lesions. Anatomical Record, 1972, 172, 298.

DE Groot, J. The rat forebrain in stereotaxic coordinates. Verhandelingen der Kiniklijke Nederlandse Akademie van Wetenschappen, Afd. Naturkunde, 1959, 52, 1-40.

Gellermans, L. W. Chance orders of alternating stimuli in visual discrimination experiments. Journal of Genetic Psychology, 1933, 42, 207-208.

Glendenning, R. L. Effects of training between two unilateral lesions of visual cortex upon ultimate retention of black-white habits by rats. Journal of Comparative and Physiological Psychology, 1972, 80, 216-229.

Goodman, D. C., \& Horel, J. A. Sprouting of optic tract projections in the brain stem of the rat. Journal of Comparative Neurology, 1966, 127, 71-88.

Hodos, W., \& BonBRIGHT, J. C. Intensity and pattern discrimination after lesions of the pretectal complex, accessory optic nucleus, and ventral geniculate in pigeons. Journal of Comparative Neurology, 1975, 161, 1-18.

Horel, J. A. Effects of subcortical lesions on brightness discrimination acquired by rats without visual cortex. Journal of Comparative and Physiological Psychology, 1968, 65, 103-109.

Horel, J. A., Bettinger, L. A., Royce, G. J., \& Meyer, D. R. Role of neocortex in the learning of two visual habits by the rat. Journal of Comparative and Physiological Psychology, 1966, 61, 66-78.

Hughes, H. C. Anatomical and neurobehavioral investigations concerning the thalamocortical organization of the rat's visual system. Journal of Comparative Neurology, 1977, 175, 311-335.

Jonason, K. R., Lauber, S., Robbins, M. J., Meyer, P. M., \& MEYER, D. R. The effects of dl-amphetamine upon discrimination performance. Journal of Comparative and Physiological Psychology, 1970, 73, 47-55.

KRIEG, W. J. S. Connections of the cerebral cortex. A: Topography of the cortical area; B: Structure of the Cortical Area. Journal of Comparative Neurology, 1946, 84, 221-323.

LASHLEY, K. S. The mechanism of vision XII. Nervous structures concerned in the acquisition and retention of habits based on reactions to light. Comparative Psychological Monographs, 1935, 11, 43-79.

LEGG, C. R., \& CowEy, A. The role of the ventral lateral geniculate nucleus and posterior thalamus in intensity discrimination in rats. Brain Research, 1977, 123, 261-273.

LEVER, T. E., \& MorLock, G. W. Nature of visual recovery following posterior neodecortication in the hooded rat. Journal of Comparative and Physiological Psychology, 1973, 83, 62-67.

Melton, A. W. The end-spurt in memorization curves as an artifact of averaging of individual curves. Psychological Monographs, 1936, 47, 119-134.

Meyer, D. R. Access to engrams. American Psychologist, 1972, 27, 124-133.

Meyer, P. M., \& MeYer, D. R. Neurosurgical procedures with special reference to the aspiration lesions. In R. B. Myers (Ed.), Methods in neurobiology. New York: Academic Press, 1971.

MEYer, D. R., \& MEYeR, P. M. Dynamics and bases of recoveries of functions after injuries to the crebral cortex. Physiological Psychology, 1977, 5, 133-165.

Meyer, P. M., Yutzey, D. A., Dalby, D. A., \& Meyer, D. R. Effects of simultaneous septal-visual, septal-anterior, and anterior-posterior lesions upon relearning a black-white discrimination. Brain Research, 1968, 8, 281-290.

Thompson, R., \& BRYANT, J. H. Memory as affected by activity of the relevant receptor. Psychological Reports, 1955, 1, 393-400.

Thompson, R., \& Rich, I. Differential effects of posterior thalamus lesions on retention of various visual habits. Journal of Comparative and Physiological Psychology, 1963, 56, 60-65.

(Received for publication May 17, 1978; revision accepted September 1, 1978.) 